\title{
Lowering backward Raman and Brillouin scattering in waveguide Raman wavelength converters
}

\author{
Min Châu Phan Huy ${ }^{1}$, Philippe Delaye ${ }^{1}$, Gilles Pauliat ${ }^{1}$, Nicolas Dubreuil ${ }^{1}$, Frédéric Gérôme ${ }^{2}$, Benoît Debord², \\ Fetah Benabid ${ }^{2}$ and Sylvie Lebrun ${ }^{1 *}$ (D)
}

\begin{abstract}
Background: Liquid filled hollow core fiber Raman wavelength converters are devices enabling to red-shift the wavelength of laser beams delivered by either fixed wavelength or tunable lasers. Most of the reported converters operate in a pulsed regime with pulse durations comprised between a few hundreds of picoseconds and up to a few nanoseconds, and energy of a few micro-Joules. However, and depending on the pulse duration, the optimization of Raman converters turns to be rather challenging since the desired forward Raman scattering may compete with counter-propagating nonlinear effects like Raman and Brillouin back-scatterings. The Brillouin back-scattering is especially hard to cancel since the Brillouin gains are usually larger than the Raman gains. Techniques have already been investigated but are not fully satisfactory. Our aim in this paper is to propose a technique to optimize the Raman forward scattering and to minimize these back-scattering effects.
\end{abstract}

Methods: We compare the interaction lengths in the forward and backward directions in the above-mentioned temporal regimes. If the fiber length is higher that the backward effective length of interaction then the backward effects should decrease at the benefit of the forward Raman scattering. Numerical simulations enable to estimate more precisely how long the fiber should be to get large Raman conversion efficiency and negligible Brillouin scattering.

Results and discussion: To validate this technique we build two identical Raman converters differing only by the fiber lengths $(0.5 \mathrm{~m}$ and $1.5 \mathrm{~m})$. We present the experimental setup. The experimental results are confronted to the numerical simulations. As expected increasing the fiber length strongly decreases the Brillouin scattering.

Conclusions: We have proposed a simple and efficient solution to minimize Brillouin back-scattering in Raman wavelength converters by optimizing the fiber length. The experimental demonstration was conducted with low energy $(\approx 6 \mu \mathrm{J})$ but the energies should scale with the fiber effective area if more energy is needed, without affecting the conversion efficiencies.

Keywords: Stimulated Raman scattering, Wavelength converters, Stimulated Brillouin scattering

\section{Background}

\section{Raman wavelength converters}

Raman wavelength converters, also known as frequency shifters, are reliable and low cost devices to red-shift the wavelength of laser beams delivered by either fixed wavelength or tunable lasers [1-3]. The input beam to be converted is simply focused inside the Raman medium. It first

\footnotetext{
* Correspondence: sylvie.lebrun@institutoptique.fr

${ }^{1}$ Laboratoire Charles Fabry, Institut d'Optique, CNRS, Univ Paris-Sud, 2 av. A.

Fresnel, 91127 Palaiseau cedex, France

Full list of author information is available at the end of the article
}

experiences spontaneous Raman scattering which inelastically scatters the incident laser beam photons into lower energy photons referred to as Stokes photons [4]. Some of these initially scattered and red-shifted photons are further amplified at the expense of the pump beam by Stimulated Raman Scattering (SRS) as they propagate through the Raman medium. These red-shifted photons form the Stokes beam. The energy difference coincides with an optical phonon assisted transition, specific of the Raman medium. In gases, liquids or crystals Raman scattering is characterized by discrete transitions. Generally 
only a few number of Raman transitions with large enough cross-sections can be exploited.

In this paper, we focus on Raman converters based on optical fibers. Conversely to Raman converters that use a laser beam focused in a crystal, a gas or a liquid cell, the interaction length achieved in a waveguide based Raman converter can be much longer than the Rayleigh length, improving the conversion efficiency. Such waveguide converters are well adapted for low power operation combined with moderate Raman cross-sections as the weak Raman conversion efficiency per unit length can be easily compensated at the expense of the fiber length. This length has to remain smaller than the effective fiber length limited by the propagation losses [5].

In order to obtain an efficient interaction of a guided light with a gas or liquid Raman medium, several approaches have been studied. The most common one employs hollow core fibers filled with a Raman liquid or gas $[2,3,6,7]$. The large variety of liquids and gases allows selecting the Raman transition over a very large span and up to $3400 \mathrm{~cm}^{-1}$ in case of water and up to $4155 \mathrm{~cm}^{-1}$ in case of hydrogen. The gas or liquid thus constitutes the active Raman material, while the fiber structure, most often silica, only contributes to the light guiding. Refractive index guiding inside the liquid core was demonstrated in various structures: high refractive index liquids in a silica tubing $[6,8,9]$; liquids in low refractive index fluorocarbon tubings [7]; liquids in the hollow core of a microstructured fiber whose cladding is composed of silica and air holes to decrease the cladding refractive index [3]. All the Raman converters based on these liquid filled hollow core capillary tubes operate under a spatially multimodal propagation. The brightness of the converted beam is thus limited by this multimodal behavior.

In case of liquids or gases whose refractive indices are lower than the one of silica, one can rely either on photonic bandgap hollow core fibers or on inhibited coupling hollow core fibers $[10,11]$. The microstructured cladding of these fibers allows light guiding even if the refractive index of the liquid or of the gas filling the holes is lower than the one of silica. Various types of wavelength converters were built either with gases or liquids $[2,12,13]$. A salient property of these converters is that, most often, single spatial mode propagation can be achieved for both the pump and the Stokes beams. The laser beam to be converted is injected at one end of the fiber, exciting one spatial guided mode (see Fig. 1). Along the propagation, amplified spontaneous Raman scattering effect leads to the generation of a copropagating Stokes beam that exits the converter, with a speckle free single mode shape. As an illustration, Raman conversion of frequency doubled micro-lasers operating at $\lambda_{\mathrm{p}}=532 \mathrm{~nm}$ into various red-shifted wavelengths $\lambda_{\mathrm{S}}$ $(556 \mathrm{~nm} ; 561 \mathrm{~nm} ; 582 \mathrm{~nm} ; 595 \mathrm{~nm} ; 612 \mathrm{~nm} ; 630 \mathrm{~nm}$; $650 \mathrm{~nm} ; 667 \mathrm{~nm} ; 772 \mathrm{~nm}$ ) were demonstrated in [14].

Lastly one can cite Raman converters realized in silica nanofibers immersed in a liquid for which the evanescent aisles of the guided mode efficiently interact with the surrounding liquid $[15,16]$.

Up to now, most of the reported liquid filled hollow core fiber Raman converters operate in a pulsed regime with pulse durations comprised between a few hundreds of picoseconds and up to a few nanoseconds, and energy of a few micro-Joules. Such pulse duration ranges are longer than the Raman relaxation times, typically a few tens of picoseconds, implying a stationary Raman excitation regime with a maximum Raman gain. However, and depending on the pulse duration, the optimization of Raman converters turns to be rather challenging since the desired forward Raman scattering may compete with counter-propagating nonlinear effects like Raman and Brillouin back-scatterings. The Brillouin back-scattering is especially hard to cancel since the Brillouin gains are usually larger than the Raman gains. Our aim in this paper is to propose a technique to optimize the Raman forward scattering and to minimize these back-scattering effects.

The article is organized as follows. In a first section the techniques that have been used to reduce backward Brillouin scatterings are presented. By stressing their incompatibility with liquid filled fiber converters, a novel technique based on a careful optimization of the fiber length is theoretically analyzed in the next section. In the last section, we present an experimental validation of this technique by building two Raman converters differing only by their length. By a proper choice of the fiber length, we show that the Brillouin scattering is strongly reduced, leading to the optimization of the Raman conversion.

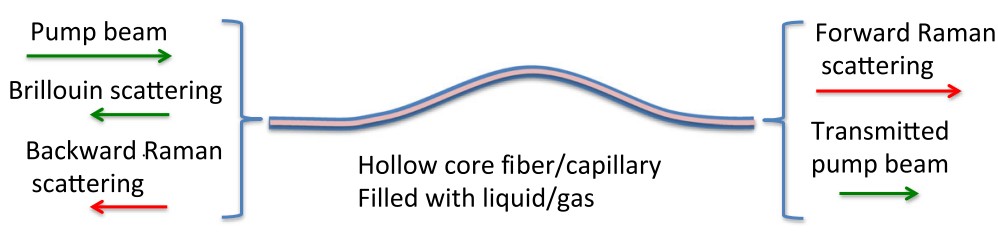

Fig. 1 Scheme of a hollow core fiber wavelength converter 


\section{Reduction of the backward Brillouin scattering}

We define the Raman conversion efficiency as the ratio of the "output forward Raman pulse energy" to the "incident pump pulse energy". Although large conversion efficiencies were already obtained in some specific cases, Raman back-scattering and especially Brillouin back-scattering may strongly limit the efficiency of the forward Raman conversion (Fig. 1). When too strong, these deleterious back-scattering effects can even prevent the correct operation of the Raman converter. To illustrate that problem, we just have to mention that many liquids are already used in the nanosecond regime for their Brillouin efficiency. For instance, alcohol based Brillouin cells have been reported for their high efficiency in the nanosecond regime without reporting any observation of a Brillouin reduction due to Raman conversion [17]. To stress this fact, we can just compare the steady-state Brillouin gain coefficient, $g_{B}$, with the forward Raman gain coefficient, $g_{R}^{f}$, in case of ethanol at the pump wavelength of $532 \mathrm{~nm}$ (derivation of numerical values are detailed in the Additional file 1):

$$
g_{B}=8010^{-12} \mathrm{~m} \cdot \mathrm{W}^{-1} \text { and } g_{R}^{f}=2.910^{-12} \mathrm{~m} \cdot \mathrm{W}^{-1}
$$

Even if we take into account the slight reduction of the Brillouin gain coefficient in the nanosecond regime compared to its value for continuous beams, Brillouin scattering should largely surpass Raman scattering, which is the exact opposite of what is required for a Raman converter!

Because of its deleterious effect for wavelength conversion, various techniques were already proposed and demonstrated to decrease Brillouin back-scattering. Most of them do not alter Raman forward scattering. The main families of techniques were listed in [18]. Without attempting to be exhaustive, we completed this list below:

1. Brillouin reduction by short pulses [19]. This technique relies on the fact that Brillouin relaxation times are much longer than Raman relaxation times. For pulse durations shorter than the Brillouin relaxation time, but longer than the Raman relaxation time, the Raman effect is unaffected while Brillouin scattering is considerably reduced.

2. Brillouin reduction by broadening the pump source $[17,20]$. Brillouin gain is reduced by using pump linewidths larger than Brillouin linewidths. Narrow linewidth lasers are thus enlarged by the use of phase modulators in order to reduce the Brillouin gain.

3. Brillouin reduction by broadening the Brillouin spectrum [21-23]. This technique was designed for fiber Raman converters. By applying a variable strain along the fiber, the Brillouin spectrum also varies which decreases Brillouin back-scattering. Alternatively, the use of segmented fibers was demonstrated.

4. Brillouin reduction by enlarging the effective Brillouin area $[18,24]$. This technique is also specific to fibers. The fiber can be designed with large acousto-optic effective areas, and thus low Brillouin gains, without compromising its optical properties.

5. Attenuation of the Brillouin Stokes wave [25]. Bragg gratings written inside the fiber can stop and increase the losses for the Brillouin back-scattered beam while remaining transparent for the forward propagating pump pulses.

6. Brillouin reduction by lowering the Brillouin gain. This technique is more specific to liquid Raman materials. Indeed, the magnitude of the Brillouin gain is inversely proportional to the Brillouin linewidth, which is itself proportional to the liquid viscosity. Increasing the liquid viscosity can be readily achieved by lowering the temperature of the liquid [26, 27]. For instance, cooling the temperature of liquid from $40^{\circ} \mathrm{C}$ down to $10^{\circ} \mathrm{C}$ was demonstrated to lead to a two-fold increase of the Brillouin threshold. Alternatively, at a given temperature, the Raman liquid can be diluted in another liquid of higher viscosity [28]. This dilution, and the resulting viscosity increase, results in a large decrease of the Brillouin gain that can largely compensate the effect of Raman gain decrease due to the lower concentration of Raman active molecules in the mixture.

All these techniques proved to be efficient. However the first technique imposes a strict constraint on the pulse duration of the pump laser. The second is more adapted to telecommunication wavelengths for which fast phase modulators are available. Techniques 3 to 5 are specific to silica fibers. Thus, only the 6th solution remains for liquid filled fiber converters. It is however not fully satisfactory. This is the reason why we investigate in this paper a new technique consisting in increasing the length of the fiber converters.

\section{Methods \\ Competition between forward and backward scatterings Procedure for optimizing forward Raman efficiency}

Our technique to optimize forward Raman scattering is relatively simple. Our starting point is to take into account the fact that both the pump and the forward Raman scattering propagate in the same direction. We are interested in the temporal regime of pulses of a few 10 ps up to a few ns, and with fiber lengths up to a few meters. The temporal propagation delay between the pump and Stokes beams (at $532 \mathrm{~nm}$ and $630 \mathrm{~nm}$ for instance) due to dispersion is thus much lower than the 
pulse duration. Temporal walk-offs can thus be neglected. Therefore the interaction length for copropagating effects is equal to the fiber length $L$. On the contrary the backward scattered beams are initiated by the leading edge of the pump beam and interact until its falling edge. Therefore, for these counter-propagating beams, the interaction length is limited by the pump pulse length to:

$$
L_{e f f} \approx \operatorname{Min}\left(L_{\Delta t} \approx \frac{c \Delta t}{2 n}, L\right)
$$

$c$ being the celerity of light in vacuum, $\Delta t$ the temporal full-width at half-maximum of the pump pulse.

Therefore, increasing the fiber length above $L_{\Delta t}$ should preferentially favor the desired forward Raman scattering over the backward Raman scattering and Brillouin scattering.

Our goal in the following is to estimate the efficiency of this technique and to determine how long the fiber should be to get a large Raman conversion efficiency and a negligible Brillouin scattering. We thus first study the evolution of the Raman conversion efficiency using a well known analytic approach [29]. The idea is to estimate the evolution of the Raman efficiency with the fiber length and pump power. We then include the back-scattering effects using numerical simulations.

\section{Forward Raman scattering neglecting backward scattering}

Presently, we neglect Raman and Brillouin backward scatterings. We also neglect all transient effects for pulses longer than the relaxation times of the involved optical Raman transitions, that is for pulses that are typically longer than about 10 ps. We thus consider that the pump pulse can be spliced in temporal slices during which the Raman gain coefficient is equal to its value for a continuous beam. We can thus exploit the extensive analyzes of forward Raman scattering presented in the literature and developed for quasi-continuous beams [5].

A convenient parameter to scale a Raman converter is the critical parameter $\gamma_{C}$ that corresponds to the value $\gamma_{C}$ of $\gamma=g_{R} I_{P} L_{\text {eff }}$ for which the output Raman Stokes intensity $I_{R}$ equals the transmitted pump beam intensity [29]. In this expression $g_{R}$ is the Raman gain coefficient, $I_{P}$ the input pump beam intensity and $L_{\text {eff }}$ the effective interaction length. In case of the forward Raman scattering we are interested in this study, $L_{e f f}=L, L$ being the fiber length.

Calculation of $\gamma_{C}$ is detailed in the literature [29]. We have plotted in Fig. 2 the value of $\gamma_{C}$ versus the gainlength product in case the Raman medium is ethanol at the pump wavelength of $532 \mathrm{~nm}$. It is noticeable that for a $g_{R}^{f} L / A_{\text {eff }}^{R}$ product spanning over 5 orders of magnitude, the critical parameter changes by a factor of two only. A

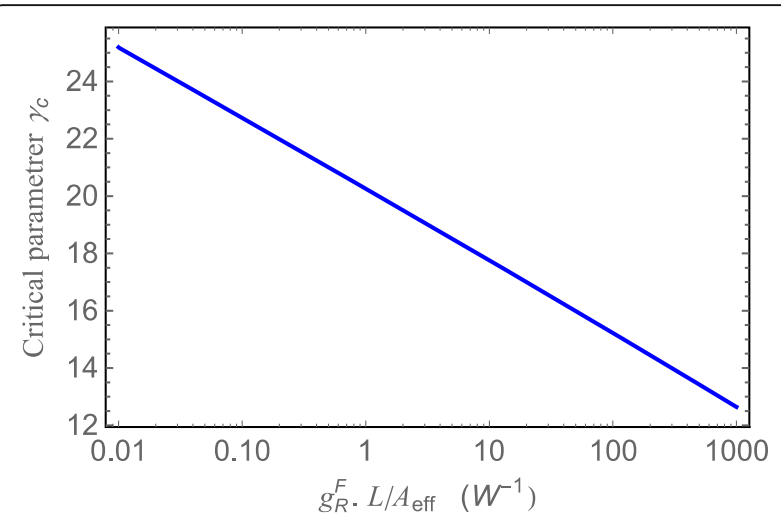

Fig. 2 Critical parameter versus the "gain $x$ effective length » product. In this case the Raman medium is ethanol pumped at $532 \mathrm{~nm}$

thumb rule to start designing a Raman converter is to state that $\gamma_{C} \approx 20$. We can also express this feature in terms of pump power required to reach the critical parameter, $P=\gamma_{C} A_{\text {eff }}^{R} /\left(g_{R}^{f} L\right)$ : increasing the fiber length by a factor $10^{5}$ and decreasing the pump power by a factor $2 \times 10^{5}$ allows keeping the same Raman conversion efficiency.

In conclusion, for smaller fiber length changes, we infer a very simple rule: a decrease of the pump beam power is compensated by an increase of the fiber length by the same amount in order to keep the forward Raman conversion efficiency constant.

We expect the influence of the fiber length to be different for back-scattering effects, because the interaction length of counter-propagating beams is limited to $L_{\text {eff }}$ by the pulse length (eq. 2). We thus anticipate that the same decrease of the pump beam power cannot be compensated by an increase of the fiber length by the same amount. To assess this prediction, we rely on the following numerical analysis taking into account forward and backward effects.

\section{Coupled-wave equations}

In the temporal regime we are interested in, with pulses larger than a few tens of picoseconds, we treat the Raman effect in the quasi-cw regime. We write the couple-wave equations for Raman and Brillouin scatterings assuming that: the pump beam is Fourier transformed limited; all beams propagate under a single spatial mode. We are not interested in the noise statistics and thus neglect beam phase fluctuation. Our simulations will thus not be valid before stimulated scattering superseded spontaneous scattering that is at very low pump powers. Furthermore, because of the absence of optical resonant cavities, all scattering processes occur at the maximum of the gain curves so that we can write all equations with real values [30]. $A_{B} A_{B}, A_{R}^{b}$ and $A_{R}^{f}$ respectively represent the modules of the slowly 
varying pump beam amplitude, Brillouin amplitude, backward and forward Raman beam amplitudes. We use normalized values so that $\|A\|^{2}$ directly represent the powers. All these amplitudes depend on time $t$ and on space coordinate $z$ along the fiber. They do not have any dependence over the transverse coordinates because we assume the beams to be single spatial modes. The transverse dependences are thus taken into account by introducing effective areas. In these equations, we consider that the effective area for the Raman process, $A_{e f f}^{R}$, may differ from the effective area for the Brillouin process, $A_{\text {eff }}^{B}[18]$.

In this set of equations, we do not consider Brillouin scattering of the Raman Stokes waves. We indeed never observed it in our experiments. That is consistent with the fact that the spectral bandwidth of the Stokes waves is much larger than the Brillouin bandwidth. We rely on references $[5,30]$ to write down these equations:

$$
\left\{\begin{aligned}
\frac{\partial A_{P}}{\partial z}+\frac{1}{v_{g}} \frac{\partial A_{P}}{\partial t} & =-\frac{g_{B}}{2 A_{e f f}^{B}} A_{B} Q-\frac{1}{2 A_{e f f}^{R}} \frac{\lambda_{S}}{\lambda_{P}}\left(g_{R}^{f}\left\|A_{R}^{F}\right\|^{2}+g_{R}^{b}\left\|A_{R}^{b}\right\|^{2}\right) A_{P} \\
\frac{\partial A_{B}}{\partial z}-\frac{1}{v_{g}} \frac{\partial A_{B}}{\partial t} & =-\frac{g_{B}}{2 A_{e f f}^{B}} A_{P} Q \\
\frac{2}{\Gamma_{B}} \frac{\partial Q}{\partial t} & =-\left(Q-Q_{0}\right)+A_{P} A_{B} \\
\frac{\partial A_{R}^{f}}{\partial z}+\frac{1}{v_{g}} \frac{\partial A_{R}^{f}}{\partial t} & =\frac{g_{R}^{f}}{2 A_{e f f}^{R}}\left(A_{R}^{f}-R\right)\left\|A_{P}\right\|^{2} \\
\frac{\partial A_{R}^{b}}{\partial z}-\frac{1}{v_{g}} \frac{\partial A_{R}^{b}}{\partial t} & =-\frac{g_{R}^{b}}{2 A_{e f f}^{R}}\left(A_{R}^{b}-R\right)\left\|A_{P}\right\|^{2}
\end{aligned}\right.
$$

$\lambda_{S}$ represents the wavelength of the Stokes wave. We neglect the difference in wavelengths between the pump and Brillouin waves, both being equal to $\lambda_{P}$. $\Gamma_{B}$ is the acoustic damping rate. Variable $Q$ is proportional to the amplitude of the acoustic wave. We normalized it in order to express the equations versus the steady state Brillouin gain $g_{B}$ [30].

In the equation for $Q$, we have neglected the space derivative, $\partial Q / \partial z$, as the acoustic velocity $v_{a}$ is much lower than the light group velocity $v_{g}[4]$. For simplicity we assume $v_{g}$ to be the same for all wavelengths, $v_{g}=c / n$ with $n$ the effective refractive group index and $c$ the speed of light in vacuum.

$g_{R}^{b}$ and $g_{R}^{f}$ are the backward and forward Raman gain coefficients that may differ, especially in gases. Indeed, $g_{R}^{b}$ can be significantly lower than $g_{R}^{f}$ due to the Doppler shift [31]. They are about the same in liquids.

Raman and Brillouin effects start from noises uniformly distributed along the fiber. These noises are formally introduced here as coefficients $R$ and $Q_{0}$. The expression for coefficient $R$ is given in the Additional file 1. $Q_{0}, A_{e f f}^{R}$ and $A_{\text {eff }}^{B}$ are the only fitting parameters in this set of equations. They are adjusted to best fit the measurements reported later on.
All simulations conducted in this article are done assuming an ethanol filled fiber. For this simulation, we selected parameters that are consistent with the experimental demonstration reported below. We give all details on the derivation of these coefficients in the Additional file 1. They are:

$\lambda_{P}=532 \mathrm{~nm} ; \lambda_{S}=630 \mathrm{~nm} ; \rho=789 \mathrm{~kg} . \mathrm{m}^{-3} ; v_{a}=$ $1205 \mathrm{~m} . \mathrm{s}^{-1} ; n=1.36 ; A_{\text {eff }}^{R}=200 \mu \mathrm{m}^{2} ; A_{\text {eff }}^{B}=1000 \mu \mathrm{m}^{2}$; $g_{R}^{f}=g_{R}^{b}=2.910^{-12} \mathrm{~m} . \mathrm{W}^{-1} ; g_{B}=8010^{-12} \mathrm{~m} . \mathrm{W}^{-1} ; \Gamma_{B}=$ 4.34 $10^{9} \mathrm{~s}^{-1} ; R=1.810^{-4} \mathrm{~W}^{1 / 2} ; Q_{0}=1.3 \mathrm{~W}$.

It is worth noting that our normalized parameter $Q$ is related to the normalized parameter $S$ of reference [32] by $S=Q A_{\text {eff }}^{B} / g_{B}$ so that $Q_{0}=1.3 \mathrm{~W}$ corresponds to $S_{0}=$ $0.1 \mathrm{~m}^{-1}$. This value is higher by a factor of about 100 than those cited by [32] for solid. Such a higher value is expected because the noise term is expected to scale as $\Gamma_{B} / v_{a}^{2}$ which is indeed about 100 times larger in liquids compared to solids [33].

\section{Numerical simulations}

Evolution of the backward Raman scattering In the undepleted pump beam approximation, Raman forward and backward scatterings evolve exponentially [29] with the exponential factor being respectively $g_{R}{ }^{f} L P / A_{\text {eff }}^{R}$ and $g_{R}^{b} L_{e f f} P / A_{\text {eff }}^{R}$. Because significant forward amplification is obtained around the critical parameter $\gamma_{c}=g_{R}^{f} L P / A_{e f f}^{R} \approx$ 20 , for which the amplification factor is $\exp (20) \approx 10^{8}$, only a small relative difference between $g_{R}^{b} L_{\text {eff }}$ and $g_{R}^{f} L$ may lead to a nearly total vanishing of the backward Raman scattering compared to the forward scattering. In gases for which, due to the Doppler shift, $g_{R}^{b}$ can be significantly lower than $g_{R}^{f}$, backward scattering may be automatically suppressed considering that $L_{\text {eff }}$ is bounded by $L$. In liquids, the two forward and backward Raman gains are about the same. To minimize backward scattering, one should lower the effective length $L_{\text {eff }}$ compared to the actual length $L$.

This is fully confirmed by the simulations and by the experiments. For $L \geq L_{\text {eff }}$, we never observed significant Raman back-scattering, for both experiments and simulations. Therefore we do not discussed anymore the Raman back-scattering in this paper.

Analysis of competition between Raman forward and Brillouin backward scattering For Brillouin scattering, as the gain is much higher, elimination of the backward signal is less evident and we will rely on numerical simulations.

In Fig. 3, we plotted an example of the numerical resolution of the set of eqs. (3). The temporal shape of the 


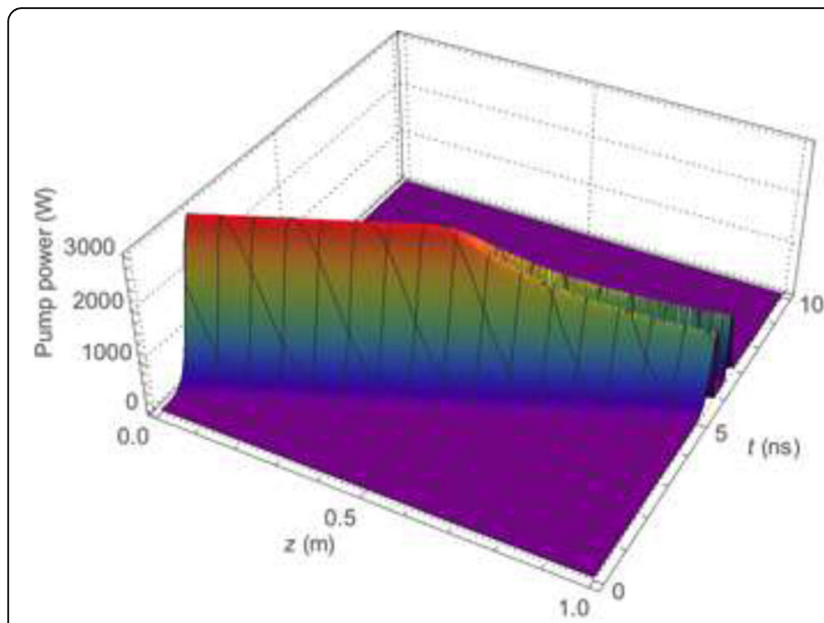

a)

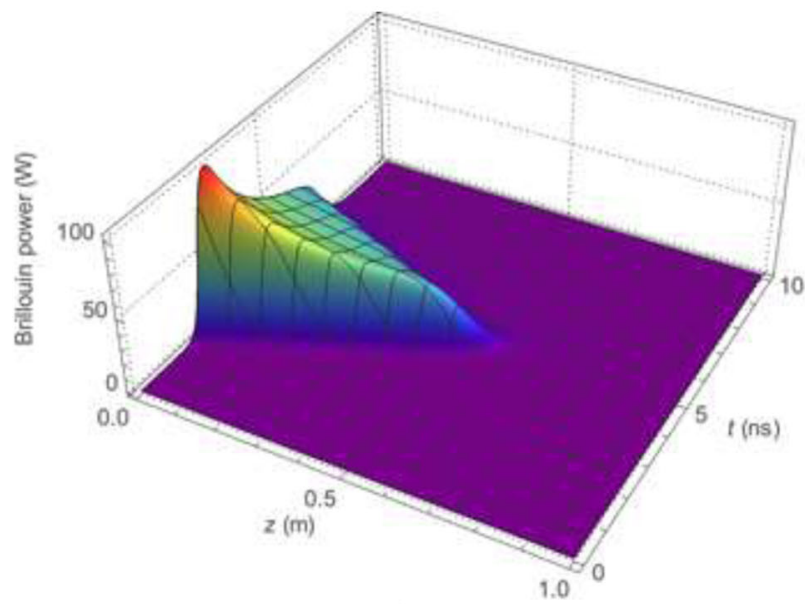

c)

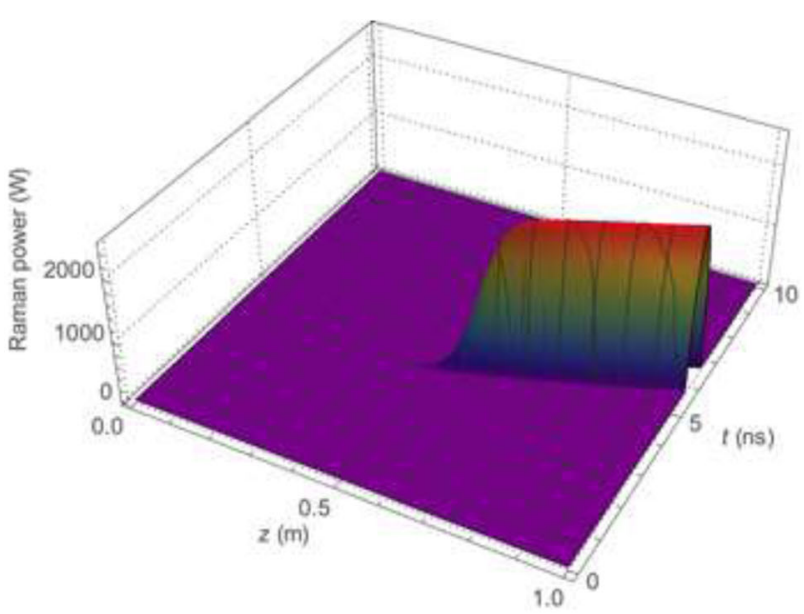

b)

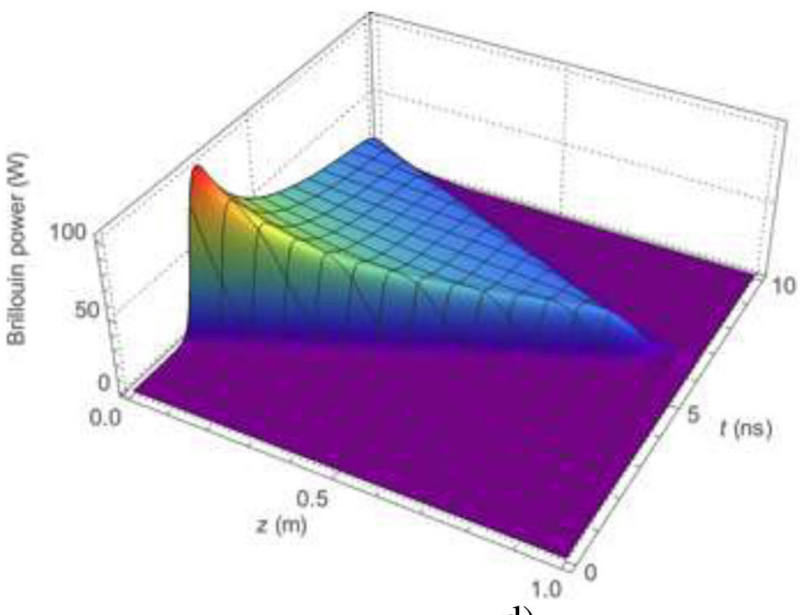

d)

Fig. 3 Evolutions of the powers versus time and coordinate $z$ for a fiber length $L=1$ m: a pump power; b Raman power; $\mathbf{c}$ Brillouin power; $\mathbf{d}$ Brillouin power in case the Raman gain is set to 0

pump beam power is Gaussian with a full-width at halfmaximum of the power, $\Delta t$, equal to $\Delta t \approx 0.9$ ns. The peak power is $P_{\text {peak }} \approx 3000 \mathrm{~W}$ and the fiber length is $L=1 \mathrm{~m}$. Because the temporal shapes of the output beam change as a function of the interaction length, we rely on values integrated over the pulse durations to define the conversion efficiencies. The Raman conversion efficiency and the Brillouin conversion efficiency are thus expressed as:

$$
\begin{aligned}
& \eta_{R}=\frac{\text { Transmitted Raman energy }}{\text { Incident pump energy }} \text {, and } \\
& \eta_{B}=\frac{\text { Reflected Brillouin energy }}{\text { Incident pump energy }}
\end{aligned}
$$

In Fig. 3a we represented the pump beam during its propagation through the fiber from $z=0 \mathrm{~m}$ to $z=L=$ $1 \mathrm{~m}$. It is strongly depleted by the forward Raman beam shown in Fig. 3b). This strong depletion is in accordance with the value of $\gamma \approx 21.6>\gamma_{C}$ at the peak of the pump beam. Brillouin back-scattered beam is represented in Fig. 3c. For these parameters, forward Raman scattering prevails over backward Brillouin scattering: $\eta_{R}=56 \%$ while $\eta_{B}=10 \%$. We observe that the Brillouin beam is significant only when the Raman beam is negligible. In order to confirm this competition between Raman and Brillouin scatterings for the same pump beam, we also plotted in Fig. 3d the backward Brillouin beam in case the Raman gain is set to zero. Although the peak power of the Brillouin scattered beam is not slightly modified, around $100 \mathrm{~W}$, the Brillouin pulse lasts longer. Consequently, the Brillouin efficiency is enlarged to $\eta_{B}=13 \%$.

Evolution of the Raman forward and Brillouin backward scatterings versus fiber length In order to evidence the evolution of the beam power as a function of the fiber 
length, we conducted a series of simulations for a constant $\gamma=g_{R}^{f} P_{\text {Peak }} L / A_{\text {eff }} \approx 21.6$ product and by varying the fiber length $L$. The results are summarized in Fig. 4. If the Brillouin gain is set to 0 , Raman efficiency $\eta_{R}$ is represented by the dotted black line. As anticipated by Fig. 2, the Raman efficiency is nearly independent of the fiber length because $\gamma$ is kept constant. When Brillouin scattering is present, the Raman efficiency is depressed (red dashed line) for the benefit of the Brillouin scattered energy. Similarly, when the Raman effect is absent, Brillouin efficiency $\eta_{B}$ is represented by the brown dot-dashed line. This Brillouin efficiency is slightly depressed in presence of the Raman effect as shown by the full blue line.

This figure thus illustrates the principle of Brillouin suppression in fiber Raman converters:

- for short lengths, Brillouin scattering predominates because the effective length of the Brillouin and Raman effects are comparable and because of the large gain for Brillouin scattering;

- for long lengths, the saturation of the effective length for Brillouin scattering favors the competition to the benefit of the Raman conversion process.

\section{Results and discussion}

\section{Experimental validation}

We validate our proposal for Brillouin suppression by comparing the importance of Brillouin scattering using two identical Raman converters differing only by the fiber lengths: $L=0.5$ mand $L=1.5 \mathrm{~m}$. They are made of a micro-structured Kagome fiber [34]. These fibers were

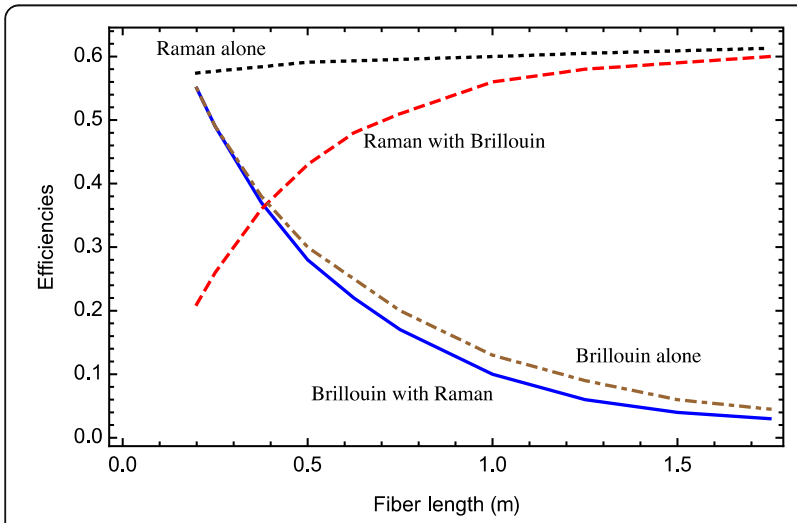

Fig. 4 Simulations of the Raman and Brillouin efficiencies versus the fiber length for a constant $\gamma=g_{R}^{f} P_{\text {Peak }} L / A_{\text {eff }}$ product. The dashed red line corresponds to the Raman conversion efficiency taking into account Brillouin effect. The blue full line corresponds to the Brillouin conversion efficiency taking into account Raman effect. The dotted black line corresponds to the Raman conversion efficiency if the Brillouin gain is set to 0 . The brown dot-dashed line stands for Brillouin efficiency in case the Raman gain is zero designed for transmitting in the near infrared when being empty. In Fig. 5 we plotted the transmission spectra of an empty fiber (red dashed line) and of a filled fiber (blue full line). The transmission spectrum is shifted towards short wavelengths when filling with ethanol, as expected [14]. Both the pump and Stokes wavelengths, $532 \mathrm{~nm}$ and $630 \mathrm{~nm}$, lay on the top of the spectrum. We have thus neglected linear losses at these two wavelengths in the simulation in accordance with the hypotheses used in eqs. (3). The second Stokes order, at $772 \mathrm{~nm}$, is also within the transmission band of these filled fibers but suffers higher losses. In the experiments, we indeed observed the appearance of this second Stokes order for high energy pulses. Nevertheless, for sake of simplicity, we did not include this second order in the simulations. All experiments are conducted at room temperature, around $20^{\circ} \mathrm{C}$. Once filled with ethanol, the fibers are hermetically sealed with one glass window at each end.

The experimental set-up is depicted in Fig. 6. The pump laser is a frequency-doubled micro-laser at $\lambda_{p}=$ $532 \mathrm{~nm}$ delivering $900 \mathrm{ps}$ pulses at a repetition rate of $4.5 \mathrm{kHz}$. Within these experimental conditions the effective backward interaction length is $10 \mathrm{~cm}$. A first lens on the laser beam path is used to compensate for the beam divergence. Then a glass filter, BG38 from Schott, blocks the unwanted remaining $1064 \mathrm{~nm}$ and $810 \mathrm{~nm}$ wavelengths emitted by the laser. The laser power is controlled by the combination of half-wave plate and polarizing beam splitter cube, PBS. The set of two mirrors, M1 and M2, is used to optimize the laser injection inside the Kagome fiber. This injection is performed by a $\times 2.5$ microscope objective, Obj1. Light from the Kagome fiber is collected by a $\times 10$ microscope objective, Obj2. A removable mirror, M3, is set either to image the spatial modal structure onto a CCD camera, or to send the output beam to the Optical Spectrum

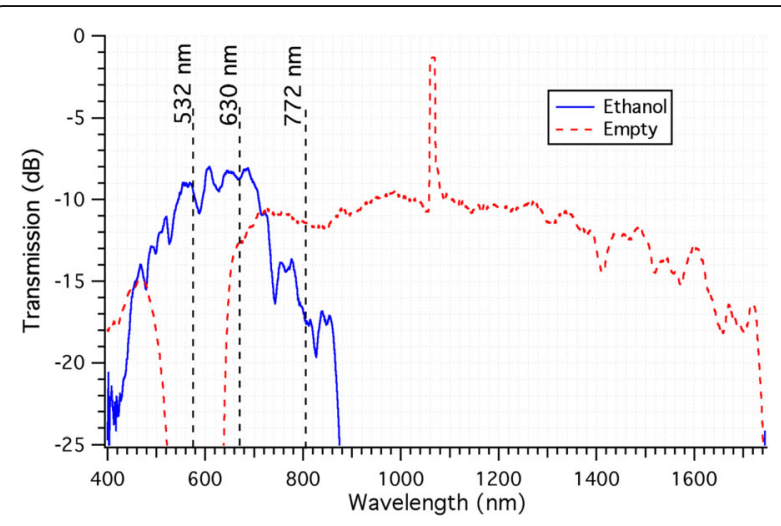

Fig. 5 Transmission spectra (not normalized) of the Kagome fiber. Red dashed line corresponds to an empty fiber; blue full line corresponds to a fiber filled with ethanol 


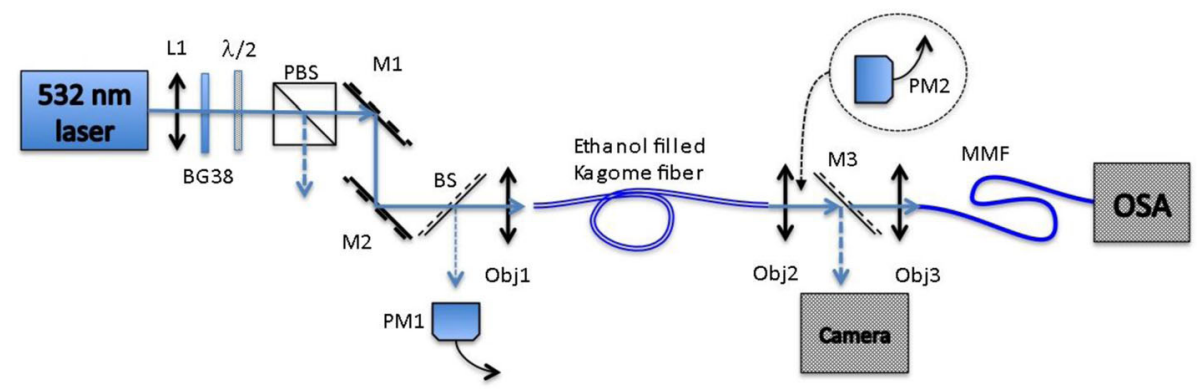

Fig. 6 Sketch of the experimental set-up, details are in the text

Analyzer, OSA, through a $\times 20$ microscope objective, Obj3, and a multimode fiber, MMF.

The measuring system $(\times 20$ microscope objective + multimode fiber + OSA) is calibrated by comparing the signal measured on the OSA with the signals measured after the $\times 10$ microscope objective with a power meter, PM2, preceded by a narrowband filter at either $772 \mathrm{~nm}$, $630 \mathrm{~nm}$ or $532 \mathrm{~nm}$.

A beam splitter, BS, set right before the microscope objective extracts part of the reflected power to measure the Brillouin power by means of another power meter, PM1. The experimental results are presented in Fig. 7.

We never observed Raman back-scattering in these two experiments, in accordance with our predictions for these fibers whose length is longer than the pulse length.

It is worth noting that we cannot experimentally measure the injected energy directly. Indeed we cannot use the cut back method for measuring the fiber losses because of the presence of the liquid: cutting the fiber requires refilling the fiber and new alignments. We thus estimated the injected energy by assuming, at low power and before the appearance of the nonlinear effects, that the only losses are the injection losses and the Fresnel losses onto the glass windows. The absorption and propagation losses along the fibers are thus assumed to be negligible so that, at low power, the injected energy is said to be equal to the pump beam transmitted energy. This simplifying assumption is reasonable given that the ethanol absorption at $532 \mathrm{~nm}$ is very small (about $\alpha \approx 0.07 \mathrm{~m}^{-1}$ [35]). The total injection losses plus Fresnel losses are thus estimated to be $29 \%$ and $33 \%$ for the two fiber lengths $L=0.5$ mand $L=1.5 \mathrm{~m}$.

When filled with ethanol, these fibers are not singlemode at our wavelengths. Our results are thus slightly sensitive to which fiber modes the pump is injected and on how the fiber is held (typically straight or wound fiber). To estimate these fluctuations, we repeated the same measurements after repositioning the optical fiber. The position of the measured critical point varies by about $10 \%$, which absolutely does not affect the conclusions reported below.

Although the fibers are not single-mode, we nevertheless checked that the pump beam remains polarized after propagating through the fiber with a polarization ratio larger than 90:1. The polarization ratio of the forward Raman beam is worse and was measured equal to about 5:1. We thus do not expect our numerical model summarized by the set of eqs. (3) to be fully accurate. Typically, although the core radius is $22 \mu \mathrm{m}$, we have conducted our simulation by assuming that the effective area is the same for the pump and Stokes beams and
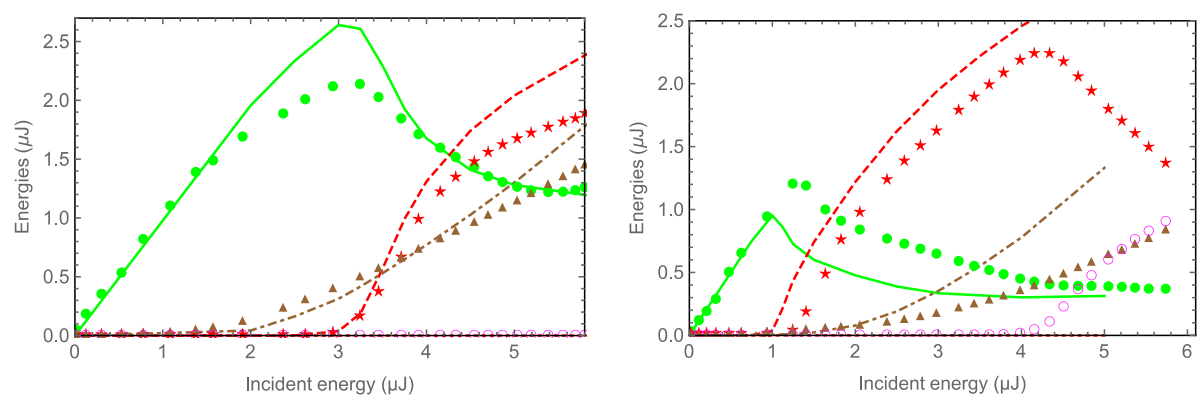

Fig. 7 Experimentally measured output energies versus the incident pump energy. The green disks correspond to the transmitted pump, the red stars correspond to the forward Raman and the brown triangles correspond to the reflected Brillouin. The lines correspond to the simulations (see text). Left figure is for a fiber length of $L=0.5 \mathrm{~m}$ and the right one is for $L=1.5 \mathrm{~m}$ 
equal to $A_{e f f}^{R} \approx 200 \mu \mathrm{m}^{2}$ that would correspond to a beam radius of $8 \mu \mathrm{m}$ if the propagation were single mode. This relatively low value for the effective area is consistent with the observation of the mode field patterns, which are smaller than the core area. This effective area can nevertheless be viewed as a fitting parameter. The acoustic area is set to $A_{\text {eff }}^{B}=1000 \mu \mathrm{m}^{2}$. These effective areas and the noise term $Q_{0}$ are the only fitting parameters in our simulations. In Fig. 7, the simulations for the transmitted pump, the forward Raman and the reflected Brillouin are represented respectively by the full green line, the dashed red line and the dot-dashed brown line.

It is noticeable that the simulations reasonably reproduce the experiments, but the second Stokes order that is not included in the model. This agreement supports our proposal to improve the Raman forward scattering at the expense of Brillouin scattering by increasing the fiber length. The various experimentally measured energies indeed evolve with the fiber length as predicted. When the fiber length is increased by a factor 3 , the Raman threshold is decreased by the same factor as anticipated (so that parameter $\gamma=g_{R}^{f} P_{\text {Peak }} L$ remains approximately the same).

As expected, increasing the fiber length strongly decreases the Brillouin back-scattering.

1) This can be noticed for instance at the critical point:

- for $L=0.5 \mathrm{~m}$, Brillouin back-reflected energy is 66\% of the output forward Raman energy, or equivalently $\eta_{R} \approx 27 \%$ and $\eta_{B} \approx 19 \%$;

- for $L=1.5 \mathrm{~m}$, Brillouin back-reflected energy is only 9\% of the output forward Raman energy.

2) For the same incident energy, for instance $4 \mu \mathrm{J}$, the longest fiber presents by far the largest Raman efficiency, about $\eta_{R} \approx 55 \%$, compared to $\eta_{R} \approx 9 \%$ for the shortest fiber.

It is worth noting that the efficiencies depend on the intensities only and not on the fiber area. The same efficiencies should be obtained whatever the mode diameter provided that the pump intensity remains the same. Therefore, the energies should scale with the fiber effective area if more energy were needed. This scaling should not affect the Raman and Brillouin conversion efficiencies.

\section{Conclusions}

We have proposed a simple solution to minimize Brillouin back-scattering in Raman wavelength converters. As illustrated by Fig. 4, for the same active medium, we can move from an operating regime at low fiber length in which Brillouin scattering strongly dominates a vanishing Raman forward scattering to a regime at large fiber length in which Raman scattering is strongly favored. The transition fiber length between these two regimes depends on the ratio of the Brillouin gain to the Raman gain and is also strongly influenced by the pulse length $\mathrm{L}_{\Delta \mathrm{t}} \approx \mathrm{c} \Delta \mathrm{t} / 2 \mathrm{n}$. In the experiment reported above the pulse length is about $10 \mathrm{~cm}$ and should theoretically strongly favour the Brillouin scattering. With a fiber length $\mathrm{L}=0.5 \mathrm{~m}$ slightly higher, we are in the transition regime with competition between Brillouin and Raman scatterings. We were for instance unable to excite the second Stokes Raman order at $772 \mathrm{~nm}$ because Brillouin scattering too strongly depletes the pump beam. With a fiber length of $\mathrm{L}=1.5 \mathrm{~m}$, that is well above the pulse length, we were able to strongly favour Raman scattering at the expense of Brillouin scattering as evidenced by the excitation of the Raman line at $772 \mathrm{~nm}$. This technique should also be efficient as well for other waveguide Raman converters, even if the exact optimum length may depend on parameters of the experiments (pump laser characteristics, used liquid, waveguide geometry, ...). Its main limitation is the inherent propagation losses of these wavelength converters, which limit the waveguide length.

\section{Additional file}

Additional file 1: Raman and Brillouin parameters [36-42]. (DOC 489 kb)

Acknowledgements

The authors thanks the "Fondation Coopération Scientifique Campus Paris Saclay" and the European ActPhast program for supporting the work of MCPH.

Funding

This work was not supported by any specific funding.

Availability of data and materials

The datasets supporting the conclusions of this article are included within the article and its additional file.

Authors' contributions

All the authors made contribution to this work. GP made the numerical simulations and wrote the article. PD and SL provided their technical and theoretical expertises on Raman converters. MCPH made the experimental measurements. ND provided its expertise on Brillouin diffusion. BD, FG and FB designed and fabricated the Kagomé fibers. All the authors read and approved the final manuscript.

Competing interests

The authors declare they have no competing interests.

\section{Publisher's Note}

Springer Nature remains neutral with regard to jurisdictional claims in published maps and institutional affiliations.

\section{Author details}

'Laboratoire Charles Fabry, Institut d'Optique, CNRS, Univ Paris-Sud, 2 av. A. Fresnel, 91127 Palaiseau cedex, France. GPPMM Group, Xlim Research Institute, CNRS UMR 7252, University of Limoges, Limoges, France.

Received: 25 September 2017 Accepted: 17 October 2017

Published online: 30 October 2017

\section{References}

1. Cerny, P., Jelinkova, H., Zverev, P.G., Basiev, T.T.: Solid state lasers with Raman frequency conversion. Prog. Quantum Electron. 28, 113-143 (2004) 
2. Benabid, F., Knight, J.C., Antonopoulos, G., Russell, P.: Stimulated Raman scattering in hydrogen-filled hollow-Core photonic crystal fiber. Science. 298, 399-402 (2002)

3. Yiou, S., Delaye, P., Rouvie, A., Chinaud, J., Frey, R., Roosen, G., Viale, P., Février, S., Roy, P., Auguste, J.L., Blondy, J.M.: Stimulated Raman scattering in an ethanol core microstructured optical fiber. Opt. Exp. 13, 4786-4791 (2005)

4. Boyd, RW: Stimulated Raman and Rayleigh-Wing Scattering. In: Nonlinear Optics (3 $3^{\text {rd }}$ edition), pp.473-510. Academic Press, Elsevier, Amserdam (2007)

5. Agrawal, GP: Stimulated Raman scattering. In: Nonlinear Fiber Optics ( $4^{\text {th }}$ edition), pp. 274-322. Academic Press, Elsevier, New-York (2010)

6. Chraplyvy, A.R., Bridges, T.J.: Infrared generation by means of multiple-order stimulated Raman scattering in $\mathrm{CCl} 4$ and $\mathrm{CbrCl} 3$-filled hollow silica fibers. Opt. Lett. 6, 632-633 (1981)

7. Altkorn, R., Koev, I., Van Duyne, R.P., Litorja, M.: Low-loss liquid-core optical fiber for low-refractive-index liquids: fabrication, characterization, and application in Raman spectroscopy. Appl. Opt. 36, 8992-8998 (1997)

8. Ippen, E.P.: Low-power quasi-cw Raman oscillator. Appl. Phys. Lett. 16, 303 (1970)

9. Stone, J.: Cw Raman fiber amplifier. Appl. Phys. Lett. 26, 163 (1975)

10. Birks, T., Roberts, P., Russell, P., Atkin, D., Shepherd, T.: Full 2-d photonic bandgaps in silica/air structures. IEEE Electron. Lett. 31, 1941-1943 (1995)

11. Couny, F., Benabid, F., Roberts, P.J., Light, P.S., Raymer, M.G.: Generation and photonic guidance of multi-octave optical-frequency combs. Science. 318, 1118 (2007)

12. Lebrun, S., Delaye, P., Frey, R., Roosen, G.: High-efficiency single-mode Raman generation in a liquid-filled photonic bandgap fiber. Opt. Lett. 32, 337-339 (2007)

13. Phan Huy, MC, Delaye, P, Pauliat, G, Debord, B, Gérôme, F, Benabid, F and Lebrun, S: Stimulated Raman scattering with large Raman shifts with liquid core Kagome fibers. Paper presented at the EOS Annual Meeting 2014, Sep 2014, Berlin, Germany. <hal-01069915>

14. Lebrun, S, Phan Huy, MC, Delaye, P and Pauliat, G: Efficient stimulated Raman scattering in hybrid liquid-silica fibers for wavelength conversion. Proc. SPIE 10021, Optical Design and Testing VII, 1002104 (October 31 2016). doi:10.1117/12.2245431

15. Shan, L., Pauliat, G., Vienne, G., Tong, L., Lebrun, S.: Design of nanofibres for efficient stimulated Raman scattering in the evanescent field. J.Eur. Opt. Soc. 8, 13030 (2013)

16. Shan, L., Pauliat, G., Vienne, G., Tong, L., Lebrun, S.: Stimulated Raman scattering in the evanescent field of liquid immersed tapered nanofibres. Appl. Phys. Lett. 102, 201110 (2013)

17. Schiemann, S., Ubachs, W., Hogervorst, W.: Efficient temporal compression of coherent nanosecond pulses in a compact SBS generator-amplifier setup. IEEE J. Quantum Electron. 33, 358-366 (1997)

18. Kobyakov, A., Sauer, M., Chowdhury, D.: Stimulated Brillouin scattering in optical fibers. Adv. Opt. Photon. 2, 1-59 (2010)

19. Su, R., Zhou, P., Wang, X., Lü, H., Xu, X.: Nanosecond pulse pumped, narrow linewidth all-fiber Raman amplifier with stimulated Brillouin scattering suppression. J. Opt. 16, 015201 (2014)

20. Willems, F.W., Muys, W., Leong, J.S.: Simultaneous suppression of stimulated Brillouin scattering and interferometric noise in externally modulated lightwave AM-SCM systems. IEEE Photon. Technol. Lett. 6, 1476-1478 (1994)

21. Yoshizawa, N., Imai, T.: Stimulated Brillouin scattering suppression by means of applying strain distribution to fiber with cabling. J. Lightw. Technol. 11, 1518-1522 (1993)

22. Zhang, L., Hu, J., Wang, J., Feng, Y.: Stimulated-Brillouin-scatteringsuppressed high-power single-frequency polarization-maintaining Raman fiber amplifier with longitudinally varied strain for laser guide star. Opt. Lett. 37, 4796-4798 (2012)

23. Engelbrecht, R:: Analysis of SBS gain shaping and threshold increase by arbitrary strain distributions. J. Lightwave Technol. 32, 1689-1700 (2014)

24. Kobyakov, A., Kumar, S., Chowdhury, D.Q., Ruffin, A.B., Sauer, M., Bickham, S.R. - Mishra, R.: Design concept for optical fibers with enhanced SBS threshold. Opt. Express. 13, 5338-5346 (2005)

25. Lee, H., Agrawal, G.P.: Suppression of stimulated Brillouin scattering in optical fibers using fiber Bragg gratings. Opt. Express. 11, 3467-3472 (2003)

26. Shi, J., Tang, Y., Wei, H., et al.: Temperature dependence of threshold and gain coefficient of stimulated Brillouin scattering in water. Appl. Phys. B Lasers Opt. 108, 717 (2012)

27. Ganot, Y., Shrenkel, S., Barmashenko, B.D., Bar, I.: Enhanced stimulated Raman scattering in temperature controlled liquid water. Appl. Phys. Lett. 105, 061107 (2014)
28. Krapchev, VB: Infrared laser system. US patent 5153887 (1991)

29. Smith, R.G.: Optical power handling capacity of low loss optical fibers as determined by stimulated Raman and Brillouin scattering. Appl. Opt. 11 2489-2494 (1972)

30. Menzel, R., Eichler, H.J.: Temporal and spatial reflectivity of focused beams in stimulated Brillouin scattering for phase conjugation. Phys. Rev. A. 46, 71397149 (1992)

31. Choi, Y.S.: Asymmetry of the forward and backward Raman gain coefficient at $1.54 \mu \mathrm{m}$ in methane. Appl. Opt. 40, 1925-1930 (2001)

32. Menzel, R: Nonlinear Interactions of Light and Matter Without Absorption. In Photonics, linear and nonlinear interactions of laser light and matter, p.198. Springer, Heidelberg (2001)

33. Boyd, R.W., Rzaewski, K., Narum, P.: Noise initiation of stimulated Brillouin scattering. Phys. Rev. A. 42, 5514 (1990)

34. Couny, F., Benabid, F., Light, P.S.: Large-pitch kagome-structured hollow-core photonic crystal fiber. Opt. Lett. 31, 3574-3576 (2006)

35. Kedenburg, S., Vieweg, M., Gissibl, T., Giessen, H.: Linear refractive index and absorption measurements of nonlinear optical liquids in the visible and near-infrared spectral region. Opt. Mater. Express. 2, 1588-1611 (2012)

36. Hellwarth, R.W.: Theory of stimulated Raman scattering. Phys. Rev. 130, 1850-1852 (1963)

37. Griffiths, J.E.: Raman-scattering cross-sections in strongly interacting liquidsystems - $\mathrm{CH} 3 \mathrm{OH}, \mathrm{C} 2 \mathrm{H} 5 \mathrm{OH}, \mathrm{I}-\mathrm{C} 3 \mathrm{H} 7 \mathrm{OH},(\mathrm{CH} 3) 2 \mathrm{CO}, \mathrm{H} 2 \mathrm{O}$, and D2O. J. Chem. Phys. 60, 2556 (1974)

38. Uchida, N.: Elastooptic coefficient of liquids determined by ultrasonic light diffraction method. Jpn. J. Appl. Phys. 7, 1259-1266 (1968)

39. Tong, J., Povey, M.J.W., Zou, X., Ward, B., Oates, C.P.: Speed of sound and density of ethanol-water mixture across the temperature range 10 to 50 degrees Celsius. J. Phys. Conf. Ser. 279, 012023 (2011)

40. Xu, J., Ren, X., Gong, W., Dai, R., Liu, D.: Measurement of the bulk viscosity of liquid by Brillouin scattering. Appl. Opt. 42, 6704-6709 (2003)

41. Nozdrev, V.F.: The viscosities of ethanol-Cyclohexane mixtures. Zh.Fiz.Khim. 49, 548-549 (1975)

42. Hirai, N., Eyring, E.: Bulk viscosity of liquids. J. Appl. Phys. 29, 810 (2016)

\section{Submit your manuscript to a SpringerOpen ${ }^{\circ}$ journal and benefit from:}

- Convenient online submission

- Rigorous peer review

- Open access: articles freely available online

- High visibility within the field

Retaining the copyright to your article

Submit your next manuscript at $>$ springeropen.com 